\title{
OPEN Effects of lipid emulsions on the formation of Escherichia coli-Candida albicans mixed-species biofilms on PVC
}

\author{
Shanshan $\mathrm{Li}^{1,2,6}$, Wanshi Duan ${ }^{3,6}$, Yujie Lei ${ }^{1}$, Zhonghui Wang ${ }^{2}$, Chaojiang Fu ${ }^{4}$, Liang He${ }^{5}$, \\ Zhenghai Shen ${ }^{1}$, Minjie $\mathrm{Li}^{1}$, Ying Chen ${ }^{1 \bowtie}$ \& Yunchao Huang ${ }^{1 \bowtie}$
}

Patients receiving lipid emulsions are at increased risk of contracting catheter-related bloodstream infections (CRBSIs) in the clinic. More than $15 \%$ of CRBSIs are polymicrobial. The objective of this study was to explore the effects of lipid emulsions on the formation of Escherichia coli (E. coli)Candida albicans (C. albicans) mixed-species biofilms (BFs) on polyvinyl chloride (PVC) surfaces and the underlying mechanism. Mixed-species BFs were produced by coculturing $E$. coli and $C$. albicans with PVC in various concentrations of lipid emulsions. Crystal violet staining and XTT assays were performed to test the mixed-species BF biomass and the viability of microbes in the BFs. The microstructures of the BFs were observed by an approach that combined confocal laser scanning microscopy, fluorescence in situ hybridization, and scanning electron microscopy. The study found that lipid emulsions could promote the formation of $E$. coli-C. albicans mixed-species BFs, especially with $10 \%$ lipid emulsions. The mechanism by which lipid emulsions promote mixed-species $B F$ formation may involve significant upregulation of the expression of the $f h D C$, iha, $H T A 1$, and $H W P 1$ genes, which are associated with bacterial motility, adhesion, and BF formation. The results derived from this study necessitate strict aseptic precautions when handling lipid emulsions and avoiding the use of high concentrations of lipid emulsions for as long as possible.

Medical devices for infusion and artificial nutrition are essentially made of plasticized polyvinyl chloride (PVC), and central venous catheters (CVCs) are no exception. CVCs are widely used in clinics to administer parenteral nutrition $(\mathrm{PN})$ due to the high risk of phlebitis ${ }^{1,2}$. However, the risk of catheter-related bloodstream infections (CRBSIs) for patients receiving a long period of PN through CVCs is relatively high due to the presence of lipid emulsions, an indispensable component of $\mathrm{PN}$, which provide vital energy and fatty acids for patients and favour the growth of various microbes, including bacteria and fungi $i^{3,4}$.

However, more than $15 \%$ of CRBSIs occurring during PN treatment are polymicrobial rather than single infections caused by one microorganism ${ }^{5}$. It is noteworthy that Candida albicans (C. albicans) is one of the leading pathogens causing CRBSIs associated with high morbidity and mortality. Approximately $27-57 \%$ of $C$. albicans infections are associated with other pathogens or opportunistic pathogens ${ }^{6,7}$. Escherichia coli (E. coli) has gradually surpassed Gram-positive bacteria as the predominant source of CRBSIs ${ }^{8,9}$. C. albicans and other pathogens can quickly colonize the surfaces of implanted medical devices and accelerate the formation of biofilms $(\mathrm{BFs})^{10}$. Once BFs form on the surfaces of indwelling medical materials, pathogenic microbes in the BFs can effectively resist immune destruction and antibiotic therapy, leading to the persistence of infections. BFs cause two-thirds of all infections. It has become clear that most BFs are polymicrobial in medical environments ${ }^{11,12}$. According

\footnotetext{
${ }^{1}$ Department of Thoracic Surgery I, Key Laboratory of Lung Cancer Research of Yunnan Province, The Third Affiliated Hospital of Kunming Medical University (Yunnan Cancer Hospital, Yunnan Cancer Center), No. 519, Kunzhou Road, Kunming 650106, China. '2Department of Anesthesiology, The Third Affiliated Hospital of Kunming Medical University (Yunnan Cancer Hospital, Yunnan Cancer Center), Kunming 650106, China. ${ }^{3}$ Department of Thoracic Surgery, Tangdu Hospital, The Second Affiliated Hospital of Air Force Medical University, Xi'an 710038, China. ${ }^{4}$ Department of Palliative Medicine, The Third Affiliated Hospital of Kunming Medical University (Yunnan Cancer Hospital, Yunnan Cancer Center), Kunming 650106, China. ${ }^{5}$ Department of Clinical Laboratory, The Third Affiliated Hospital of Kunming Medical University (Yunnan Cancer Hospital, Yunnan Cancer Center), Kunming 650106, China. ${ }^{6}$ These authors contributed equally: Shanshan Li and Wanshi Duan. ${ }^{\square}$ email: 36410008@ qq.com; huangych2001@aliyun.com
} 

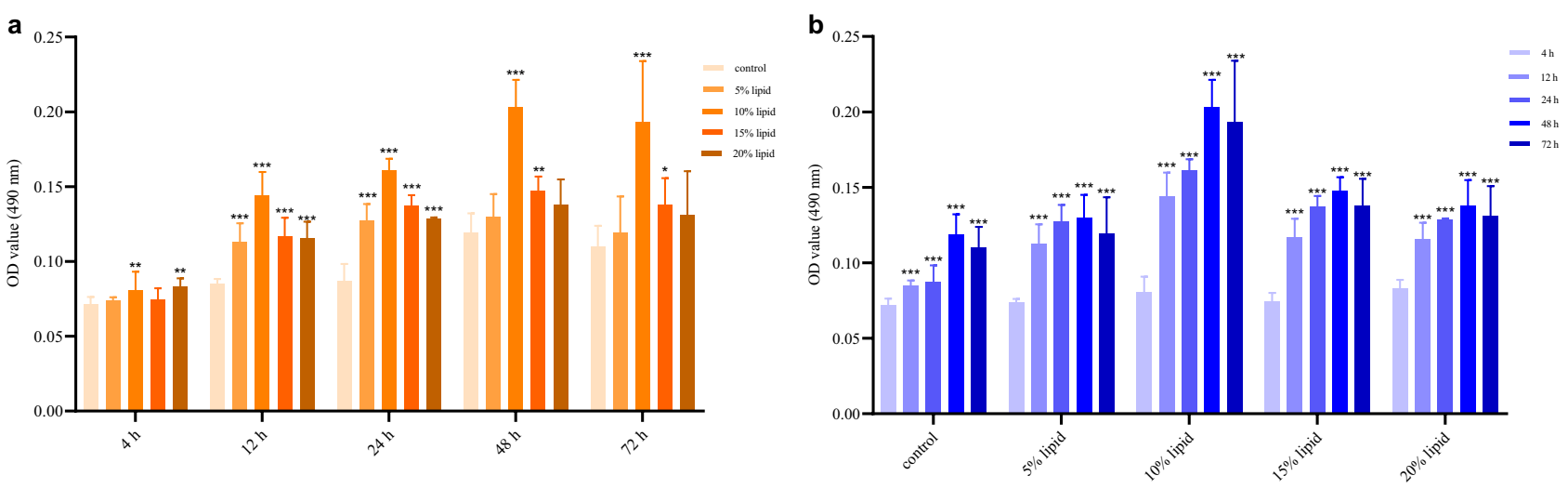

Figure 1. Effect of lipid emulsions on microbial adhesion and mixed-species BF biomass. Compared with the control group at the same time, ${ }^{\star} P<0.05,{ }^{* *} P<0.01,{ }^{* * *} P<0.001$ (a). Compared with the $4 \mathrm{~h}$ at the same concentration, ${ }^{\star} P<0.05,{ }^{* *} P<0.01,{ }^{* *} P<0.001$ (b).

to existing research, clinical biomaterial infections that were polymicrobial had a poorer clinical prognosis than single-species infections, exhibiting twice the mortality rate of single-species infections ${ }^{13,14}$. Several studies have reported that lipid emulsions are associated with an increased risk of contracting a CRBSI. Nevertheless, these studies were limited to studying infections caused by monospecies BFs ${ }^{15-17}$. Few studies have focused on the formation of mixed-species BFs on the surfaces of medical devices. The effects of lipid emulsions on the formation of E. coli-C. albicans mixed-species BFs on medical catheters are still unknown.

For the above reasons, an in vitro model of a mixed-species BF of E. coli-C. albicans was constructed on a PVC surface in the present study. We aimed to determine whether the formation of E. coli-C. albicans mixedspecies BFs was significantly altered by clinical lipid emulsions. In addition, we further explored the effects of lipid emulsions on the morphology and architecture of mixed-species BFs. The correlation of the expression of the $f l h D C$, iha, HTA 1 , and HWP1 genes with BF formation was also analysed. The flhDC gene of $E$. coli encodes transcription factors that initiate flagellar synthesis and enhance the swimming and swarming motilities of the bacterium. The iha gene encodes a pathogenic adherence-conferring outer-membrane protein in E. coli. HTA1 has been shown to play a significant role in mediating genomic rearrangement and amplification in C. albicans. HWP1 is directly associated with hypha formation and invasiveness in C. albicans.

\section{Results}

Effects of lipid emulsions on microbial adhesion and mixed-species BF biomass. After $4 \mathrm{~h}, 12 \mathrm{~h}$, $24 \mathrm{~h}, 48 \mathrm{~h}$, and $72 \mathrm{~h}$ of coculture, semiquantitative detection of microbial adhesion and BF biomass on PVC surfaces was conducted in each group. The results showed that the lipid emulsions with various concentrations exhibited greater microbial adhesion and mixed-species BF biomass than those in the control group, which was treated with tryptic soy broth (TSB) medium alone at each time point $(P<0.05)$. The $10 \%$ lipid emulsions had the most significant effect, followed by the $15 \%$ and $20 \%$ lipid emulsions. The detection results for each group from 4 to $72 \mathrm{~h}$ showed that the microbial adhesion ability and BF biomass increased with incubation time and were highest at $48 \mathrm{~h}(P<0.001)$ (Fig. 1a,b).

Effects of lipid emulsions on microbial viability in mixed-species BFs. After $4 \mathrm{~h}, 12 \mathrm{~h}, 24 \mathrm{~h}, 48 \mathrm{~h}$, and $72 \mathrm{~h}$ of coculture, the viability of microbes in mixed-species BFs on the surfaces of PVC pieces was examined by using XTT in each group. There were significant differences between the lipid emulsion groups with various concentrations and the control group at $12-72 \mathrm{~h}(P<0.05)$. The $10 \%$ lipid emulsion group showed the most significant effect of all groups, followed by the $15 \%$ and $20 \%$ lipid emulsion groups. The results showed that the microbial viability of each group increased with incubation time and was highest at $48 \mathrm{~h}(P<0.001)$ (Fig. 2a,b).

Thickness and live/dead microbes of mixed-species BFs. The formation of mixed-species BFs on the surfaces of PVC pieces was found to be a dynamic process. The thickness of the BFs in each group increased rapidly in $24 \mathrm{~h}$ of coculture and peaked at $48 \mathrm{~h}$. There was a slight decline at $48-72 \mathrm{~h}$ in each group. The mixedspecies BFs of the lipid emulsion groups were more complex and denser than those of the control group. The thickness of mixed-species BFs in the $10 \%, 15 \%$, and $20 \%$ lipid emulsion groups was thicker than the control group at $24 \mathrm{~h}, 48 \mathrm{~h}$, and $72 \mathrm{~h}(P<0.05)$ (Fig. 3a).

Both live and dead microbes in the mixed-species BFs were observed by confocal laser scanning microscopy (CLSM). The live microbes were stained green by SYTO9, whereas the dead microbes were stained red by propidium iodide (PI). At $24 \mathrm{~h}$, there were only small proportions of dead microbes in the mixed-species BFs in each group. At 48-72 h, the proportions of live microbes decreased, and the proportions of dead microbes increased gradually from the outer layers to the inner layers of the mixed-species BFs (Fig. 3b,c). 

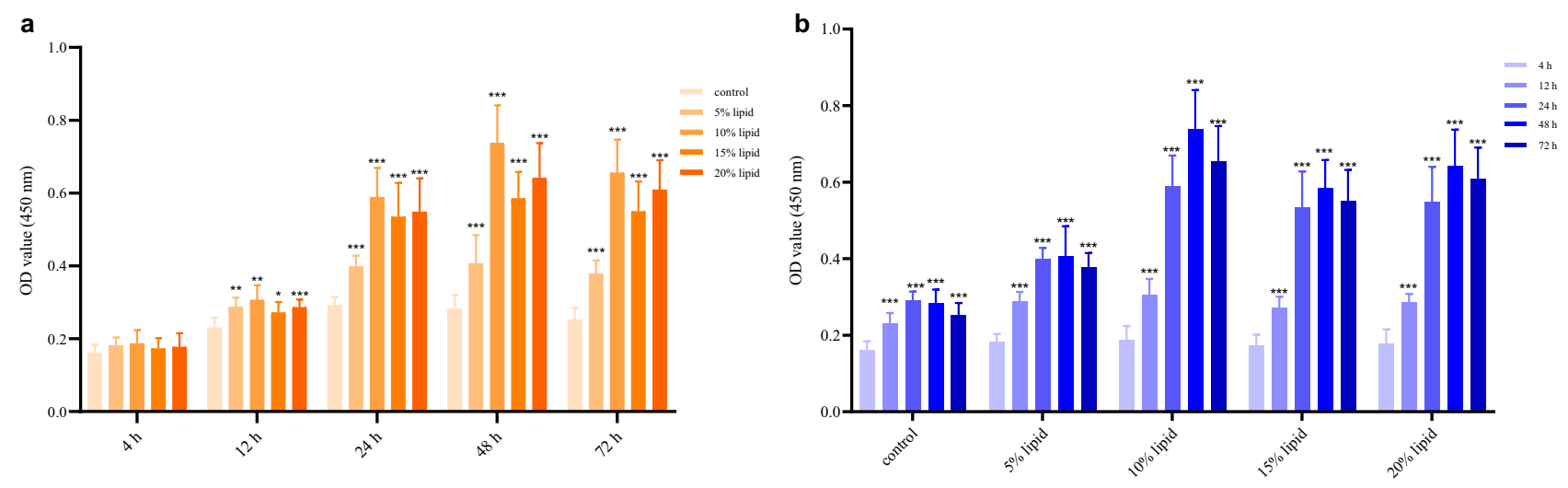

Figure 2. Effect of lipid emulsions on microbial viability in mixed-species BFs. Compared with the control group at the same time, ${ }^{\star} P<0.05,{ }^{* *} P<0.01,{ }^{\star * *} P<0.001$ (a). Compared with the $4 \mathrm{~h}$ at the same concentration, ${ }^{\star} P<0.05,{ }^{* *} P<0.01,{ }^{* *} P<0.001(\mathbf{b})$.

Composition of mixed-species BFs detected by fluorescence in situ hybridization (FISH). At $24 \mathrm{~h}$, E. coli and C. albicans grew together in the BFs and formed clumps in the control group. The structure of mixed-species BFs in the lipid emulsion groups was more complex than that in the control group. At $72 \mathrm{~h}$, the $\mathrm{BFs}$ were denser and more robust than those at $24 \mathrm{~h}$ in each group. After exposure to the $10 \%, 15 \%$, and $20 \%$ lipid emulsions for $72 \mathrm{~h}$, E. coli grew around the overlapping and interlaced C. albicans, and the mixed-species BFs looked like a "Christmas tree forest". The "Christmas tree forest"-like appearance indicates the formation of strong BFs. At $24 \mathrm{~h}$ and $72 \mathrm{~h}$, in the control group and 5\% lipid emulsion group, the proportions of C. albicans were greater than those of $E$. coli in the mixed-species BFs. The proportions of these two microbes in the mixedspecies BFs were similar in the $10 \%$ lipid emulsion group. When the concentration of lipid emulsions exceeded $15 \%$, E. coli was obviously dominant in the mixed-species BFs (Fig. 4).

Microstructure of mixed-species BFs detected by scanning electron microscopy (SEM). It was found that E. coli adhered to the chlamydospores, pseudohyphae, and mycelia of C. albicans. At $72 \mathrm{~h}$, the BFs in each group were more complex and denser than those at $24 \mathrm{~h}$, forming a three-dimensional network structure. The lipid emulsions remained a part of the mixed-species BFs and were attached to the surfaces of bacteria, mycelia and chlamydospores, forming more complex and mature BFs, especially in the $10 \%, 15 \%$, and $20 \%$ lipid emulsion groups. With increasing lipid emulsion concentrations, an increasing amount of $E$. coli was found in the mixed-species BFs. C. albicans was closely surrounded by E. coli. The growth of C. albicans was inhibited when the concentration of lipid emulsions exceeded $15 \%$. There was a significant decrease in the capacity of $C$. albicans to undergo the yeast-to-hypha switch after exposure to $10 \%, 15 \%$, and $20 \%$ lipid emulsions for $72 \mathrm{~h}$ (Fig. 5).

Gene expression by quantitative real-time polymerase chain reaction (qRT-PCR). Specific differences were noted in the expression levels of various genes between the $10 \%$ lipid emulsion group and the control group at various time points. After $24 \mathrm{~h}$ of coculture, the expression levels of the flhDC, iha, HTA1, and HWP1 genes were upregulated in the $10 \%$ lipid emulsion group compared with those in the control group. The upregulation of the iha gene was the most obvious $(P<0.05)$ (Fig. 6a). The expression of the flhDC and HWP1 genes decreased after $48 \mathrm{~h}$ of coculture. The expression of the iha gene, which exhibited slight upregulation in the $10 \%$ lipid emulsion group after $48 \mathrm{~h}$ of coculture, had a similar pattern as that of the HTA1 gene $(P<0.05)$ (Fig. 6b). After $72 \mathrm{~h}$ of coculture, the expression levels of these four genes were downregulated in the $10 \%$ lipid emulsion group compared with the control group $(P<0.05)$ (Fig. 6c).

\section{Discussion}

In tumour patients with immune dysfunction and patients who consume glucocorticoids for long time periods, the BFs formed are usually a mixture of bacteria and fungi. E. coli and C. albicans are often coisolated in cases of $\mathrm{BF}$-associated infection $\mathbf{s}^{18,19}$. Microbes reside in mixed-species BFs, in which organism growth and metabolism are different from those in monospecies $\mathrm{BF}^{20,21}$. Increased quantity and diversity of the species present increase the complexity of mixed-species BFs and enhance antimicrobial resistance ${ }^{22,23}$. The treatment of mixed-species $\mathrm{BFs}$ is more difficult and challenging than that of monospecies BFs. Therefore, research on factors influencing mixed-species BF formation has great clinical significance in reducing CRBSIs induced by lipid emulsions. Our experiments uniquely illustrated differences among various concentrations of lipid emulsions with regard to their effects on E. coli-C. albicans mixed-species BF formation. These data have the potential to impact the treatment of patients receiving PN.

In our research, PVC was used as the CVC material, and an E. coli-C. albicans mixture was used as the source of opportunistic pathogen coinfections. TSB medium was used as a supplement for various concentrations of lipid emulsions because it could support both E. coli and C. albicans ${ }^{24}$. The results of CLSM, FISH, and SEM showed that E. coli and C. albicans could coexist in TSB medium or lipid emulsions of various concentrations 

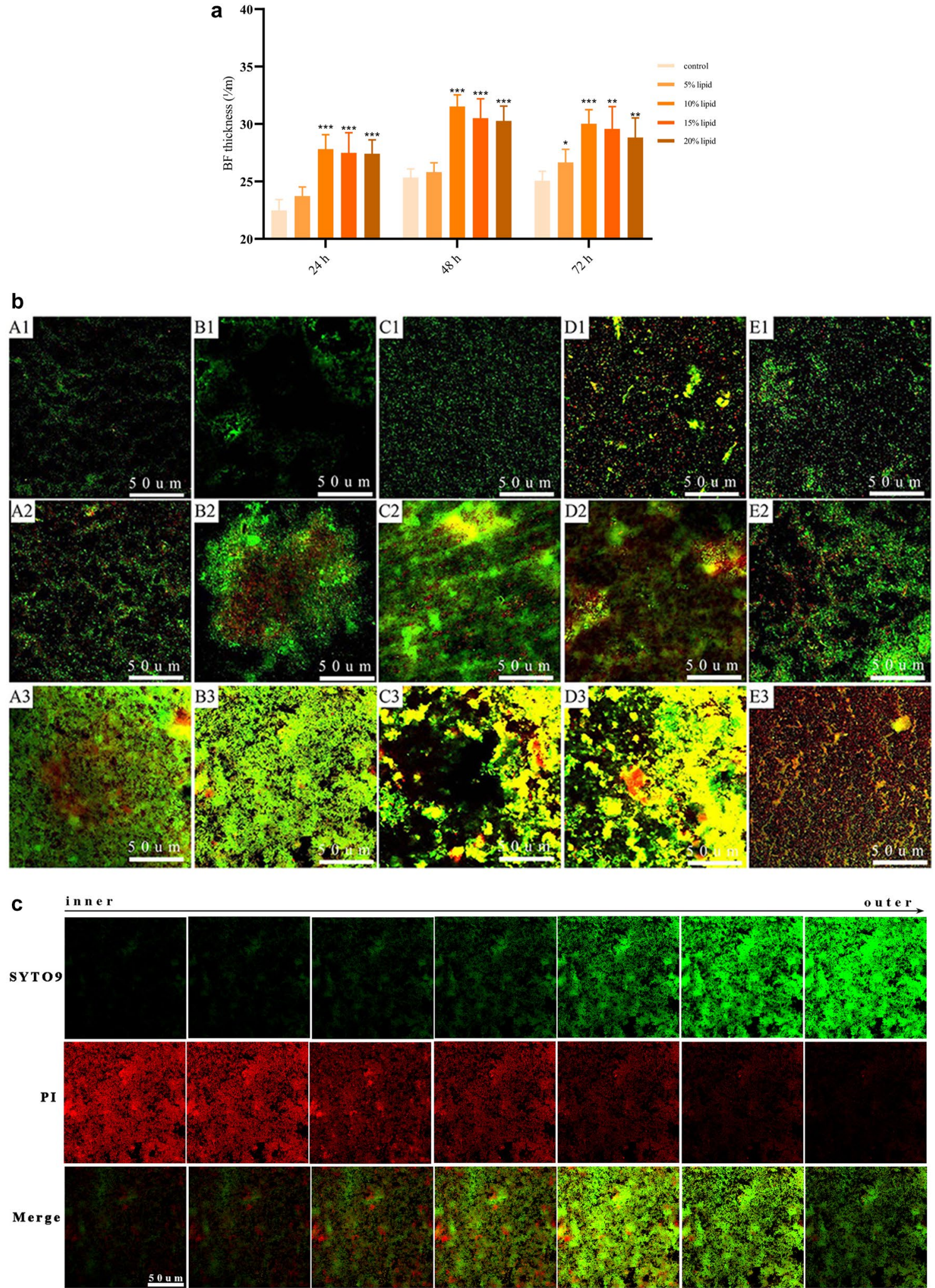

Figure 3. Thickness of mixed-species BFs at 24, 48, and $72 \mathrm{~h}$. Compared with the control group at the same time, ${ }^{\star} P<0.05,{ }^{* \star} P<0.01,{ }^{* *} P<0.001$ (a). Observation of mixed-species BFs by CLSM at 24,48 , and $72 \mathrm{~h}(\mathbf{b})$. Observation of the live/dead microbes of mixed-species BFs by CLSM (c). The images were obtained at $\times 200$ magnification. (A1) Control group (24 h); (B1) 5\% lipid emulsion group (24 h); (C1) 10\% lipid emulsion group (24 h); (D1) 15\% lipid emulsion group (24 h); (E1) 20\% lipid emulsion group (24 h); (A2) control group (48 h); (B2) 5\% lipid emulsion group (48 h); (C2) 10\% lipid emulsion group (48 h); (D2) 15\% lipid emulsion group (48 h); (E2) 20\% lipid emulsion group (48 h); (A3) control group (72 h); (B3) 5\% lipid emulsion group (72 h); (C3) $10 \%$ lipid emulsion group (72 h); (D3) $15 \%$ lipid emulsion group (72 h); (E3) $20 \%$ lipid emulsion group (72 h). 
$24 \mathrm{~h}$

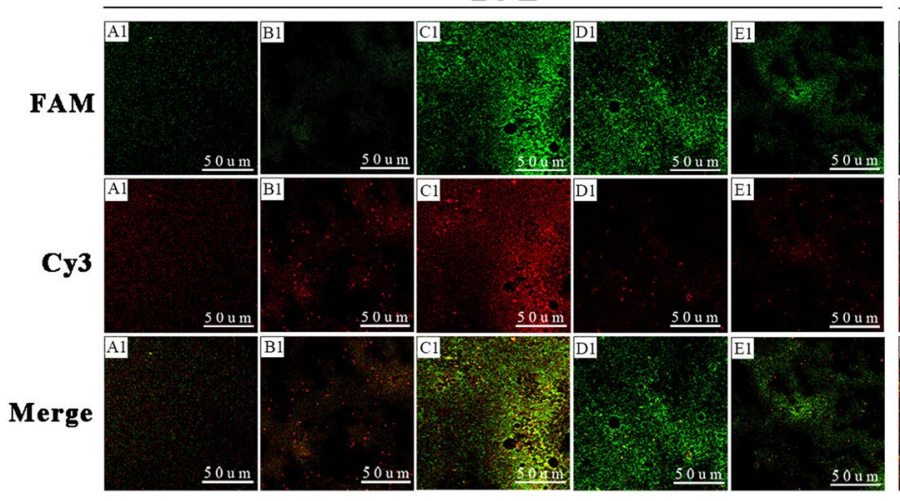

$72 \mathrm{~h}$

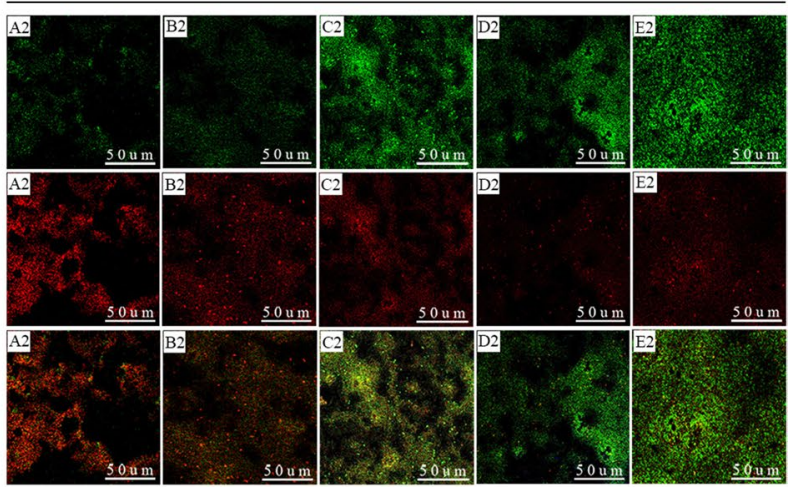

Figure 4. Observation of the composition of mixed-species BFs by FISH-CLSM at $24 \mathrm{~h}$ and $72 \mathrm{~h}$. The images were obtained at $\times 200$ magnification. (A1) Control group (24 h); (B1) 5\% lipid emulsion group (24 h); (C1) 10\% lipid emulsion group (24 h); (D1) 15\% lipid emulsion group (24 h); (E1) 20\% lipid emulsion group (24 h); (A2) control group (72 h); (B2) 5\% lipid emulsion group (72 h); (C2) 10\% lipid emulsion group (72 h); (D2) 15\% lipid emulsion group (72 h); (E2) 20\% lipid emulsion group (72 h).
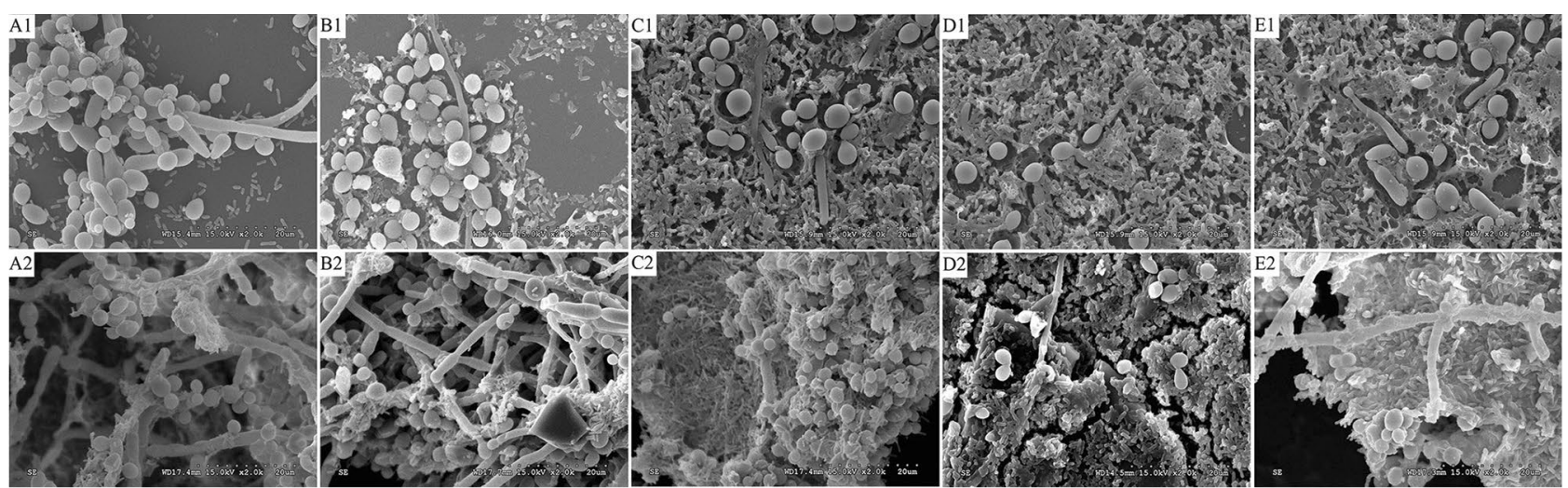

Figure 5. Observation of mixed-species BFs by SEM at $24 \mathrm{~h}$ and $72 \mathrm{~h}$. The images were obtained at $\times 2000$ magnification. (A1) Control group (24 h); (B1) 5\% lipid emulsion group (24 h); (C1) 10\% lipid emulsion group (24 h); (D1) 15\% lipid emulsion group (24 h); (E1) 20\% lipid emulsion group (24 h); (A2) control group (72 h); (B2) 5\% lipid emulsion group (72 h); (C2) 10\% lipid emulsion group (72 h); (D2) 15\% lipid emulsion group (72 h); (E2) 20\% lipid emulsion group (72 h).
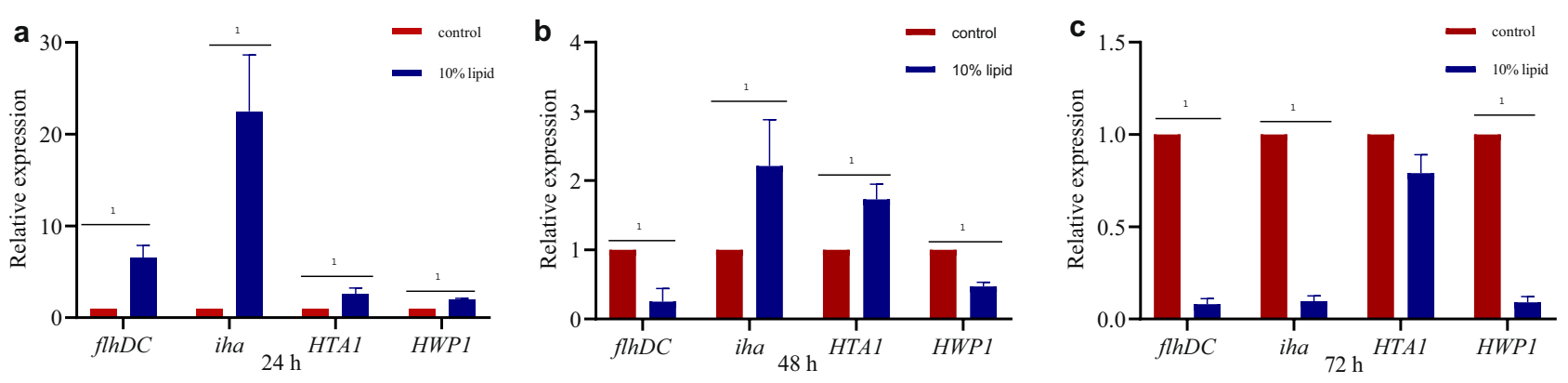

Figure 6. Comparison of qRT-PCR results of gene expression at 24,48 , and $72 \mathrm{~h}$ between the control group and the $10 \%$ lipid emulsion group, ${ }^{1} P<0.05$.

and develop mixed-species BFs on the surface of PVC. Mixed-species BFs were the main cause of coinfection with E. coli-C. albicans via implanted biomaterials. The results of crystal violet (CV) and XTT assays, and the thickness of the mixed-species BFs showed that microbial adhesion, viability, and mixed-species BF formation were promoted by lipid emulsions, and $10 \%$ was the optimum lipid emulsion concentration among the four preset concentrations for the growth and formation of E. coli-C. albicans mixed-species BFs.

In addition, we used live/dead BacLight bacterial staining and CLSM to visualize the internal structure of mixed-species BFs. This was proven to be a reliable technique for the evaluation of microbial viability in $\mathrm{BFs}^{25,26}$. 
The images revealed that the proportions of live pathogens declined gradually from the outer to inner layers due to the relatively hypoxic and nutrient-deficient environment. Dead pathogens were abundantly located at the bottom and inside of the mixed-species BFs. This was consistent with previous research ${ }^{10,27}$. Nucleic acids released into the extracellular environment by dead pathogens have been demonstrated to contribute to the formation and structural stability of BFs and provide abundant nutrients for live pathogens to support the sustainable growth of $\mathrm{BFs}^{28}$. This result suggested that over time, the number of dead cells at the bottom of the BFs increased, and the structure of the BFs became more stable and robust.

Furthermore, by SEM and FISH, we observed a novel phenomenon that wherein E. coli was predominant in the mixed-species BFs after exposure to the $15 \%$ and $20 \%$ lipid emulsions for a long time, and the growth of C. albicans was inhibited and transformed from the hypha phase to the yeast phase. However, these results did not conclusively indicate that mixed-species BF formation was impaired when the concentration of the lipid emulsions exceeded 15\% because we also observed the "Christmas tree" structure representing strong vitality in these groups. Swindell et al. pointed out that $10 \%$ Intralipid was conducive to the germination of $C$. albicans chlamydospores and the growth of mycelia, which was not entirely consistent with our experimental results ${ }^{16}$. The apparent differences between these two studies might be attributed to the BF species and different types of lipid emulsions. However, our findings are supported by other studies. These studies have proven that $C$. albicans has a unique sensitivity to medium-chain fatty acids with carbon chain lengths of $\mathrm{C} 8, \mathrm{C} 10$, and $\mathrm{C} 12$, represented by caprylic, capric, and lauric acid, respectively ${ }^{29,30}$. The abovementioned medium-chain fatty acids have been demonstrated to have antifungal effects ${ }^{31,32}$. The lipid emulsions used in our experiments contained mediumand long-chain fatty acids. The carbon chain lengths were C6-24. Moreover, the interactions between species are very complex, ranging from cooperation to competition and even predation ${ }^{33,34}$. E. coli can significantly influence candidal growth and induce hyphal death ${ }^{35}$. Interestingly, a study by Cabral et al. demonstrated that C. albicans could be killed by coculturing with $E$. coli in vitro and asserted that a soluble factor secreted by $E$. coli resulted in this $\mathrm{effect}^{36}$. However, other previous studies showed that $E$. coli could contribute to the colonization and invasiveness and the damaging effects of C. albicans ${ }^{37,38}$. These studies may help to explain the differences in the composition and architecture of mixed-species BFs grown in various concentrations of lipid emulsions. The interactions between species and the complex structure of mixed-species BFs can explain why infections caused by mixed-species BFs associated with biomaterials are extremely difficult to treat.

To better elucidate the mechanism underlying E. coli-C. albicans mixed-species BF formation enhanced by lipid emulsions, flhDC and iha gene expression in E. coli was measured. flhDC mainly regulates the biosynthesis of flagella and increases the motility and ability to colonize biomaterials ${ }^{39,40}$. The iha gene is the iron-regulated gene A homologue adhesin. The gene encodes a bacterial outer-membrane protein associated with the adhesion of bacteria $^{41}$. In the $E$. coli strains causing primitive acute pyelonephritis, iha was associated with higher BF biomass formation ${ }^{42}$. Based on the qRT-PCR results and the functions of $f l h D C$ and iha, the current study confirmed a potential role of $10 \%$ lipid emulsions in promoting the movement and colonization of $E$. coli at the early stage of BF formation. After the initial adhesion was completed, the aggregation capacity of $E$. coli was enhanced by lipid emulsions during the middle stage of BF formation. In addition, HWP1 and HTA 1 gene expression in C. albicans was measured. HWP1 encodes a fungal cell wall protein required for hyphal development and yeast adhesion to epithelial cells ${ }^{43}$. HWP1 is directly associated with BF formation in C. albicans. HTA1 encodes histone $\mathrm{H} 2 \mathrm{~A}$ of $C$. albicans and reflects the growth and reproductive ability of C. albicans cells to a certain extent ${ }^{44}$. The present study showed that HWP1 and HTA1 were expressed at a higher level in the $10 \%$ lipid emulsion group than in the control group at $24 \mathrm{~h}$, indicating that lipid emulsions further the growth and hypha formation in $C$. albicans only at the early stage of BF formation. This result showed high concordance with SEM results. After $72 \mathrm{~h}$ of coculture, the four genes were downregulated in the $10 \%$ lipid emulsion group which might be related to nutrient consumption from the medium or most of the microbes in the mature mixed-species BFs entering the dormant stage.

In summary, the effects of lipid emulsions on E. coli-C. albicans mixed-species BFs were reported for the first time in our study. We demonstrated that lipid emulsions significantly stimulated E. coli-C. albicans mixedspecies BF formation by upregulating the expression of genes related to BF formation. Avoiding the use of high concentrations of lipid emulsions for as long as possible may be helpful in reducing CRBSIs during PN. Finally, further research on the possible mechanism by which lipid emulsions promote the formation of mixed-species BFs is needed to overcome microbial infections.

\section{Methods}

Microbes, reagents, and equipment. E. coli (MC1000) was a gift from the Yale Coli Genetic Stock Center. C. albicans (ATCC10231) was purchased from the Institute of Microbiology, Chinese Academy of Sciences. PVC was purchased from Guangdong Kewei (China) Co., Ltd., processed into pieces with sizes of $5 \mathrm{~mm} \times 5 \mathrm{~mm}$ or $8 \mathrm{~mm} \times 8 \mathrm{~mm}$, and sterilized by $24 \mathrm{~h}$ of formaldehyde fumigation for further experiments. Lipid emulsions (C6-24) were obtained from Fresenius Kabi (China). The TSB medium and XTT bacterial proliferation and cytotoxicity kit were supplied by Huankai (China) Microbial Technology Co., Ltd. The total RNA extraction kit was supplied by Tiangen Biotech (China) Co., Ltd. Primers were synthesized by Sangon Biotech (China) Co., Ltd. The cDNA synthesis kit was procured from Bio-Rad (USA). The FISH kit was purchased from Boxin Biology (China) Co., Ltd. The live/dead BacLight bacterial viability kit was obtained from Life (USA). The S-3000N scanning electron microscope was from Hitachi (Japan), and the FV1000 confocal laser scanning microscope was from Olympus (Japan). qRT-PCR assays were conducted using SuperReal PreMix Plus supplied by Tiangen Biotech (China) and were performed on an ABI 7500 PCR system (USA). 


\begin{tabular}{|l|l|l|}
\hline Strain & Probe sequence & Fluorescent dye \\
\hline E. coli $(\mathrm{MC1000)}$ & $\begin{array}{l}\text { AGAGAAGCGACCTCGCGAGAGCAAGCGGACCTCAT } \\
\text { AAAGTGCGTCGTAGTCCGGATTGGAGTCTGCAACT }\end{array}$ & FAM (green) \\
\hline C. albicans $($ ATCC10231) & ACCAGACTTGCCCTCC & Cy3 (red) \\
\hline
\end{tabular}

Table 1. Primer probe sequences.

Culture of microbes and experimental grouping. Standard strains of C. albicans and E. coli were inoculated on Sarpaul agar plates and $\mathrm{MH}$ agar plates, respectively, and incubated at $37^{\circ} \mathrm{C}$ for $24 \mathrm{~h}$. Subsequently, a single colony picked from the Sarpaul agar plate or $\mathrm{MH}$ agar plate with an inoculation ring was individually inoculated into a test tube containing $5 \mathrm{~mL}$ of TSB medium. These tubes were incubated in a constant-humidity oscillator at $37^{\circ} \mathrm{C}$ and $200 \mathrm{r} / \mathrm{min}$ for $16-18 \mathrm{~h}$. After the cells grew to the logarithmic growth phase, the concentration of the microbial solution in each tube was adjusted to $1.1 \times 10^{7}$ cells $/ \mathrm{mL}$ using TSB medium in an ultraviolet spectrophotometer for later use. The mixed microbial solution in the study was prepared in a 1:1 ratio; that is, $2 \mathrm{~mL}$ of microbial solution was prepared by mixing $1 \mathrm{~mL}$ of C. albicans yeast solution and $1 \mathrm{~mL}$ of E. coli bacterial solution. The experiment included five groups: a control group and four groups with various concentrations of lipid emulsions $(5 \%, 10 \%, 15 \%$, or $20 \%)$. The TSB medium was mixed with the $20 \%$ lipid emulsions in various proportions to modulate the lipid emulsion concentration to $5 \%, 10 \%, 15 \%$, or $20 \%$. The mixed microbial solution with PVC pieces was treated with the above concentrations of lipid emulsions. As a control, samples were prepared similarly with TSB medium. All groups were incubated in a thermostatic incubator at $37^{\circ} \mathrm{C}$ with $5 \% \mathrm{CO}_{2}$.

Detection of microbial adhesion and BF biomass by crystal violet (CV) staining. Then, $100 \mu \mathrm{L}$ of the previously prepared lipid emulsions of various concentrations or TSB medium was added to a 96-well cell culture plate, and $10 \mu \mathrm{L}$ of the mixed microbial solution and a $5 \mathrm{~mm} \times 5 \mathrm{~mm}$ PVC piece were added into each well. Six wells were inoculated in each group in each plate. Plates were incubated for $4 \mathrm{~h}, 12 \mathrm{~h}, 24 \mathrm{~h}, 48 \mathrm{~h}$, and $72 \mathrm{~h}$ in an incubator at $37^{\circ} \mathrm{C}$ to allow E. coli and C. albicans to adhere to the surfaces of PVC pieces and induce mixed-species $\mathrm{BF}$ formation. After coculturing, the medium was removed from the 96-well plates, and $100 \mu \mathrm{L}$ of PBS was added to wash and remove the floating microbes on the PVC piece three times. After gently washing and discarding the PBS, the BF was fixed with $95 \%$ methanol for $30 \mathrm{~min}$. Then, $100 \mu \mathrm{L}$ of $2 \% \mathrm{CV}$ dye solution was added to each well after fixation, and the plate was incubated at $37^{\circ} \mathrm{C}$ for $30 \mathrm{~min}$. Then, the $\mathrm{CV}$ dye solution was discarded, and each well was rinsed with $100 \mu \mathrm{L}$ of PBS three times. The bound CV was resolubilized in $100 \mu \mathrm{L}$ of DMSO, and the absorbance was measured at $490 \mathrm{~nm}$ on a multifunctional marker to determine the microbial adhesion ability and quantify the biomass of BFs in the experimental wells. Wells containing TSB medium and a PVC piece were similarly processed and used as blank controls. The experiments were repeated three times independently.

Detection of microbial viability by XTT. The mixed microbial solution was cocultured with various concentrations of lipid emulsions or TSB medium for $4 \mathrm{~h}, 12 \mathrm{~h}, 24 \mathrm{~h}, 48 \mathrm{~h}$, and $72 \mathrm{~h}$ as described above and processed for the XTT assay as previously described ${ }^{45}$. Briefly, the PVC pieces in the wells were gently washed three times with cold PBS solution to remove the floating microbes on the PVC pieces. After gently washing and discarding the PBS from the 96-well plates, $100 \mu \mathrm{L}$ of TSB medium and $20 \mu \mathrm{L}$ of XTT solution were added to each well, and the plates were incubated at $37^{\circ} \mathrm{C}$ for $2 \mathrm{~h}$ in the absence of light. Following incubation, the absorbance was measured at $450 \mathrm{~nm}$ to detect the viability of microbes in BFs by using a multifunctional marker. Wells containing TSB medium and a PVC piece were similarly processed and used as blank controls. The experiments were repeated three times independently.

Observation of the thickness and live/dead microbes of mixed-species BFs by CLSM. An $8 \mathrm{~mm} \times 8 \mathrm{~mm}$ PVC piece and $100 \mu \mathrm{L}$ of mixed microbial solution were added to each well of 24 -well plates and cocultured with various concentrations of lipid emulsions $(2 \mathrm{~mL})$ in an incubator at $37^{\circ} \mathrm{C}$ in the experimental groups. As a control, the PVC pieces and mixed microbial solution were treated with only TSB medium. The fluorescent stains for testing microbial viability in the BFs were identified using a live/dead BacLight bacterial viability kit. The protocol for sample preparation for the viability assay is described in detail in the supplementary data (see S1). CLSM observation was carried out using an argon laser. The green fluorescence excitation wavelength was $488 \mathrm{~nm}$, and the emission wavelength was $519 \mathrm{~nm}$. The red fluorescence excitation wavelength was $559 \mathrm{~nm}$, and the emission wavelength was $567 \mathrm{~nm}$. The live and dead microbes on the PVC pieces were evaluated according to the area occupied by the green fluorescence of live microbes and red fluorescence of dead microbes. Each PVC piece was scanned from the inside to the outside to measure the thickness of the BF.

Observation of mixed-species BF composition by FISH. FISH was used in combination with CLSM to visualize the distribution of the two strains in the mixed-species BFs. The probe sequences of $E$. coli and $C$. albicans designed according to the sequences in GenBank are shown in Table 1. Probes for the 16S rRNA of $E$. coli and C. albicans were labelled with 5-carboxyfluorescein (FAM) and cyanine dye (Cy3), respectively. The protocol for sample preparation for FISH is described in detail in the supplementary data (see S2). After FISH and washing, the structure and composition of the mixed-species BFs on PVC pieces were observed by CLSM. 


\begin{tabular}{|l|l|l|}
\hline Target gene & Forward primer $\left(\mathbf{5}^{\prime} \mathbf{\prime} \mathbf{3}^{\prime}\right)$ & Reverse primer $\left(\mathbf{5}^{\prime} \mathbf{-} \mathbf{3}^{\prime}\right)$ \\
\hline flhDC & GCGGTTTGTTGAAAGTGGAT & GATGGCGGTTGACATAAGC \\
\hline iha & ATGATAACCGGGATGGTCAA & CCCATTTGTCGCTCTTCAGT \\
\hline $16 \mathrm{~S}$ rRNA & GAGAGCAAGCGGACCTCATA & GCAGACTCCATTCCGGACTAC \\
\hline$H T A 1$ & ATGTCAGGTGGTAAAGGTAAAG & CTACAATTCTTGAGAAGCCTTAAC \\
\hline HWP1 & GGTTGTGAGCCATTAGGGTTA & GGTTGTGAGCCATTAGGGTTA \\
\hline ACT1 & ACCACCGGTATTGTTTTGGA & TGGACAAATGGTTGGTCAAG \\
\hline
\end{tabular}

Table 2. flhDC, iha, $16 \mathrm{~S}$ rRNA, HTA1, HWP1 and ACT1 primer sequences.

The green fluorescence excitation wavelength for FAM was $488 \mathrm{~nm}$, and the emission wavelength for FAM was $519 \mathrm{~nm}$. The red fluorescence excitation wavelength for Cy3 was $559 \mathrm{~nm}$, and the emission wavelength for Cy3 was $567 \mathrm{~nm}$.-

Observation of mixed-species BF microstructure by SEM. The sample preparation method for SEM was mainly as described in the literature ${ }^{46}$. In summary, $8 \times 8 \mathrm{~mm}$ PVC pieces were removed from the 24 -well plates after incubation for $24 \mathrm{~h}$ and $72 \mathrm{~h}$ as described above, gently washed with PBS solution three times to remove the medium and floating microbes, and then fixed with $2 \%$ glutaraldehyde-containing phosphate buffer for $24 \mathrm{~h}$ at $4{ }^{\circ} \mathrm{C}$. After primary fixation, the PVC pieces were washed three times with PBS solution for $10 \mathrm{~min}$ each to remove fixatives and residual medium. The PVC pieces were then postfixed with $1 \%$ osmium tetroxide for $2 \mathrm{~h}$ at $4{ }^{\circ} \mathrm{C}$ and then dehydrated in an ethanol gradient series $(25 \%, 50 \%, 75 \%$ and $100 \%)$ for $20 \mathrm{~min}$. Then, $100 \%$ anhydrous ethanol was replaced with isoamyl acetate for $20 \mathrm{~min}$ and frozen at $-20{ }^{\circ} \mathrm{C}$ following permeation with tert-butyl alcohol at $40^{\circ} \mathrm{C}$ for $2 \mathrm{~h}$. The samples were scanned by SEM to observe the microstructure of mixed-species BFs after undergoing critical point drying with carbon dioxide $\left(\mathrm{CO}_{2}\right)$ and ion sputter coating with golden brown.

Quantitation of BF-related gene expression. A 24-well cell culture plate was removed from the control group and $10 \%$ lipid emulsion group at $24 \mathrm{~h}, 48 \mathrm{~h}$, and $72 \mathrm{~h}$, and the BFs were scraped and transferred to $1.5 \mathrm{~mL}$ centrifuge tubes. Total RNA was extracted with a total RNA extraction kit for RNA quantitation. Reverse transcription was conducted with the extracted RNA samples by the Bio-Rad iScript cDNA Synthesis Kit. Primers were designed for $f l h D C$, iha, $H T A 1$, and $H W P 1$, as well as for $16 \mathrm{~S}$ rRNA and $A C T 1$, which were used as the reference genes. The primer sequences are shown in Table 2. The primers and cDNA template synthesized from the reverse transcription reaction were used for qRT-PCR. The $2^{-\Delta \Delta C t}$ method was used for comparison of the relative levels of mRNAs ${ }^{47}$.

Statistical analysis and image construction. SPS 24.0 statistical software was used for statistical analyses. The experimental data conforming to the normal distribution or meeting the normal distribution after conversion were expressed as the mean \pm standard deviation. Analysis of variance (ANOVA) was used for intragroup and intergroup comparisons, and a $t$-test was used for pairwise comparisons. A value of $P<0.05$ was considered statistically significant. $P<0.01$ and $P<0.001$ indicated significant differences. All graphs were generated by GraphPad Prism 8.0.1 (https://www.graphpad.com). Images were converted to appropriate format and resolution using Adobe Photoshop CS6 (https://www.adobe.com).

\section{Data availability}

All the data in the paper are available.

Received: 17 February 2021; Accepted: 5 August 2021

Published online: 19 August 2021

\section{References}

1. Christensen, L. D. et al. Comparison of complications associated with peripherally inserted central catheters and Hickman ${ }^{\mathrm{m}}$ catheters in patients with intestinal failure receiving home parenteral nutrition. Six-year follow up study. Clin. Nutr. (Edinburgh, Scotland) 35, 912-917. https://doi.org/10.1016/j.clnu.2015.06.009 (2016).

2. Kim, J. S., Holtom, P. \& Vigen, C. Reduction of catheter-related bloodstream infections through the use of a central venous line bundle: Epidemiologic and economic consequences. Am. J. Infect. Control 39, 640-646. https://doi.org/10.1016/j.ajic.2010.11.005 (2011).

3. Austin, P. D., Hand, K. S. \& Elia, M. Factors influencing Escherichia coli and Enterococcus durans growth in parenteral nutrition with and without lipid emulsion to inform maximum duration of infusion policy decisions. JPEN J. Parenteral Enteral Nutr. 39, 953-965. https://doi.org/10.1177/0148607114538456 (2015).

4. Luzzati, R. et al. Peripheral and total parenteral nutrition as the strongest risk factors for nosocomial candidemia in elderly patients: A matched case-control study. Mycoses 56, 664-671. https://doi.org/10.1111/myc.12090 (2013).

5. Santarpia, L. et al. Central venous catheter related bloodstream infections in adult patients on home parenteral nutrition: Prevalence, predictive factors, therapeutic outcome. Clin. Nutr. (Edinburgh, Scotland) 35, 1394-1398. https://doi.org/10.1016/j.clnu. 2016.03.009 (2016).

6. Todd, O. A. et al. Candida albicans augments Staphylococcus aureus virulence by engaging the staphylococcal agr quorum sensing system. MBio https://doi.org/10.1128/mBio.00910-19 (2019). 
7. Sheehan, G., Tully, L. \& Kavanagh, K. A. Candida albicans increases the pathogenicity of Staphylococcus aureus during polymicrobial infection of Galleria mellonella larvae. Microbiology (Reading, England) 166, 375-385. https://doi.org/10.1099/mic.0.000892 (2020).

8. Lee, Y. M. et al. Clinical impact of delayed catheter removal for patients with central-venous-catheter-related Gram-negative bacteraemia. J. Hosp. Infect. 99, 106-113. https://doi.org/10.1016/j.jhin.2018.01.004 (2018).

9. Braun, E. et al. Predominance of Gram-negative bacilli among patients with catheter-related bloodstream infections. Clin. Microbiol. Infect. 20, O627-629. https://doi.org/10.1111/1469-0691.12565 (2014).

10. Chen, Y. et al. Study on the structure of Candida albicans-Staphylococcus epidermidis mixed species biofilm on polyvinyl chloride biomaterial. Cell Biochem. Biophys. 73, 461-468. https://doi.org/10.1007/s12013-015-0672-y (2015).

11. de la Fuente-Núñez, C., Reffuveille, F., Fernández, L. \& Hancock, R. E. Bacterial biofilm development as a multicellular adaptation: Antibiotic resistance and new therapeutic strategies. Curr. Opin. Microbiol. 16, 580-589. https://doi.org/10.1016/j.mib.2013.06. $013(2013)$.

12. Elias, S. \& Banin, E. Multi-species biofilms: Living with friendly neighbors. FEMS Microbiol. Rev. 36, 990-1004. https://doi.org/ 10.1111/j.1574-6976.2012.00325.x (2012).

13. Morales, D. K. \& Hogan, D. A. Candida albicans interactions with bacteria in the context of human health and disease. PLoS Pathog. 6, e1000886. https://doi.org/10.1371/journal.ppat.1000886 (2010).

14. Kali, A. et al. Prevalence of Candida co-infection in patients with pulmonary tuberculosis. Australas. Med. J. 6, 387-391. https:// doi.org/10.4066/amj.2013.1709 (2013).

15. Touré, A. et al. Propensity score analysis confirms the independent effect of parenteral nutrition on the risk of central venous catheter-related bloodstream infection in oncological patients. Clin. Nutr. (Edinburgh, Scotland) 32, 1050-1054. https://doi.org/ 10.1016/j.clnu.2012.12.006 (2013).

16. Swindell, K., Lattif, A. A., Chandra, J., Mukherjee, P. K. \& Ghannoum, M. A. Parenteral lipid emulsion induces germination of Candida albicans and increases biofilm formation on medical catheter surfaces. J. Infect. Dis. 200, 473-480. https://doi.org/10. 1086/600106 (2009).

17. Chheng, M. L., Heidbreder, C., Btaiche, I. F. \& Blackmer, A. B. Infectious complications with nondaily versus daily infusion of intravenous fat emulsions in non-critically ill adults. Nutr. Clin. Pract. 28, 737-744. https://doi.org/10.1177/0884533613507085 (2013).

18. Vandecandelaere, I., Matthijs, N., Nelis, H. J., Depuydt, P. \& Coenye, T. The presence of antibiotic-resistant nosocomial pathogens in endotracheal tube biofilms and corresponding surveillance cultures. Pathog. Dis. 69, 142-148. https://doi.org/10.1111/2049632X.12100 (2013)

19. Samaranayake, Y. H. et al. Enteric Gram-negative bacilli suppress Candida biofilms on Foley urinary catheters. APMIS Acta Pathol. Microbiol. Immunol. Scand. 122, 47-58. https://doi.org/10.1111/apm.12098 (2014).

20. Oxaran, V. et al. Behavior of foodborne pathogens Listeria monocytogenes and Staphylococcus aureus in mixed-species biofilms exposed to biocides. Appl. Environ. Microbiol. 84, e02038-02018. https://doi.org/10.1128/AEM.02038-18 (2018).

21. Dong, J. et al. Research on the effect of $\mathrm{TiO}(2)$ nanotubes coated by gallium nitrate on Staphylococcus aureus-Escherichia coli biofilm formation. J. Clin. Lab. Anal. 34, e23417. https://doi.org/10.1002/jcla.23417 (2020).

22. Chen, P. et al. Characterization of mixed-species biofilm formed by Vibrio parahaemolyticus and Listeria monocytogenes. Front. Microbiol. 10, 2543. https://doi.org/10.3389/fmicb.2019.02543 (2019).

23. El Kadri, H. et al. The antimicrobial efficacy of remote cold atmospheric plasma effluent against single and mixed bacterial biofilms of varying age. Food Res. Int. (Ottawa, Ont.) 141, 110126. https://doi.org/10.1016/j.foodres.2021.110126 (2021).

24. Thein, Z. M., Smaranayake, Y. H. \& Smaranayake, L. P. Dietary sugars, serum and the biocide chlorhexidine digluconate modify the population and structural dynamics of mixed Candida albicans and Escherichia coli biofilms. APMIS Acta Pathol. Microbiol. Immunol. Scand. 115, 1241-1251. https://doi.org/10.1111/j.1600-0643.2007.00735.x (2007).

25. Tawakoli, P. N., Al-Ahmad, A., Hoth-Hannig, W., Hannig, M. \& Hannig, C. Comparison of different live/dead stainings for detection and quantification of adherent microorganisms in the initial oral biofilm. Clin. Oral Investig. 17, 841-850. https://doi.org/10. 1007/s00784-012-0792-3 (2013).

26. Hu, W., Murata, K. \& Zhang, D. Applicability of LIVE/DEAD BacLight stain with glutaraldehyde fixation for the measurement of bacterial abundance and viability in rainwater. J. Environ. Sci. (China) 51, 202-213. https://doi.org/10.1016/j.jes.2016.05.030 (2017).

27. Fox, E. P. et al. Anaerobic bacteria grow within Candida albicans biofilms and induce biofilm formation in suspension cultures. Curr. Biol. 24, 2411-2416. https://doi.org/10.1016/j.cub.2014.08.057 (2014).

28. Nagasawa, R., Yamamoto, T., Utada, A. S., Nomura, N. \& Obana, N. Competence-stimulating-peptide-dependent localized cell death and extracellular DNA production in Streptococcus mutans biofilms. Appl. Environ. Microbiol. https://doi.org/10.1128/aem. 02080-20 (2020).

29. Willems, H. M. E., Stultz, J. S., Coltrane, M. E., Fortwendel, J. P. \& Peters, B. M. Disparate Candida albicans biofilm formation in clinical lipid emulsions due to capric acid-mediated inhibition. Antimicrob. Agents Chemother. https://doi.org/10.1128/aac.0139419 (2019).

30. Murzyn, A., Krasowska, A., Stefanowicz, P., Dziadkowiec, D. \& Łukaszewicz, M. Capric acid secreted by S. boulardii inhibits C. albicans filamentous growth, adhesion and biofilm formation. PLoS One 5, e12050. https://doi.org/10.1371/journal.pone.00120 50 (2010).

31. Huang, C. B., Alimova, Y., Myers, T. M. \& Ebersole, J. L. Short- and medium-chain fatty acids exhibit antimicrobial activity for oral microorganisms. Arch. Oral Biol. 56, 650-654. https://doi.org/10.1016/j.archoralbio.2011.01.011 (2011).

32. Takahashi, M., Inoue, S., Hayama, K., Ninomiya, K. \& Abe, S. Inhibition of Candida mycelia growth by a medium chain fatty acids, capric acid in vitro and its therapeutic efficacy in murine oral candidiasis. Med. Mycol. J. 53, 255-261. https://doi.org/10.3314/ mmj.53.255 (2012)

33. Jackrel, S. L., Schmidt, K. C., Cardinale, B. J. \& Denef, V. J. Microbiomes reduce their host's sensitivity to interspecific interactions. MBio https://doi.org/10.1128/mBio.02657-19 (2020).

34. Menon, R., Ramanan, V. \& Korolev, K. S. Interactions between species introduce spurious associations in microbiome studies. PLoS Comput. Biol. 14, e1005939. https://doi.org/10.1371/journal.pcbi.1005939 (2018).

35. Bandara, H. M., Cheung, B. P., Watt, R. M., Jin, L. J. \& Samaranayake, L. P. Secretory products of Escherichia coli biofilm modulate Candida biofilm formation and hyphal development. J. Investig. Clin. Dent. 4, 186-199. https://doi.org/10.1111/jicd.12048 (2013).

36. Cabral, D. J., Penumutchu, S., Norris, C., Morones-Ramirez, J. R. \& Belenky, P. Microbial competition between Escherichia coli and Candida albicans reveals a soluble fungicidal factor. Microb. Cell (Graz, Austria) 5, 249-255. https://doi.org/10.15698/mic20 18.05.631 (2018).

37. Farrokhi, Y., Al-Shibli, B., Falah-Joudah Al-Hameedawi, D., Neshati, Z. \& Makhdoumi, A. Escherichia coli enhances the virulence factors of Candida albicans, the cause of vulvovaginal candidiasis, in a dual bacterial/fungal biofilm. Res. Microbiol. https://doi. org/10.1016/j.resmic.2021.103849 (2021).

38. Yang, W., Zhou, Y., Wu, C. \& Tang, J. Enterohemorrhagic Escherichia coli promotes the invasion and tissue damage of enterocytes infected with Candida albicans in vitro. Sci. Rep. 6, 37485. https://doi.org/10.1038/srep37485 (2016).

39. Fahrner, K. A. \& Berg, H. C. Mutations that stimulate flhDC expression in Escherichia coli K-12. J. Bacteriol. 197, 3087-3096. https://doi.org/10.1128/jb.00455-15 (2015). 
40. Huang, W. C. et al. The role of the bacterial protease Prc in the uropathogenesis of extraintestinal pathogenic Escherichia coli. J. Biomed. Sci. 27, 14. https://doi.org/10.1186/s12929-019-0605-y (2020).

41. Paniagua-Contreras, G. L. et al. Comprehensive expression analysis of pathogenicity genes in uropathogenic Escherichia coli strains. Microb. Pathog. 103, 1-7. https://doi.org/10.1016/j.micpath.2016.12.008 (2017).

42. Pompilio, A. et al. Phylogenetic relationships, biofilm formation, motility, antibiotic resistance and extended virulence genotypes among Escherichia coli strains from women with community-onset primitive acute pyelonephritis. PLoS One 13, e0196260. https:// doi.org/10.1371/journal.pone.0196260 (2018).

43. Orsi, C. F. et al. Impact of Candida albicans hyphal wall protein 1 (HWP1) genotype on biofilm production and fungal susceptibility to microglial cells. Microb. Pathog. 69-70, 20-27. https://doi.org/10.1016/j.micpath.2014.03.003 (2014).

44. Tenea, G. N. et al. Overexpression of several Arabidopsis histone genes increases Agrobacterium-mediated transformation and transgene expression in plants. Plant Cell 21,3350-3367. https://doi.org/10.1105/tpc.109.070607 (2009).

45. Koban, I. et al. XTT assay of ex vivo saliva biofilms to test antimicrobial influences. GMS Krankenhaushygiene interdisziplinar 7 , Doc06. https://doi.org/10.3205/dgkh000190 (2012).

46. Deleon, H. 3rd. Application of polystyrene disk substrates in cellular cultivation methods: Generalized specimen preparation protocol for scanning electron microscopy. J. Microsc. 264, 189-197. https://doi.org/10.1111/jmi.12429 (2016).

47. Li, C. et al. MKRN3-mediated ubiquitination of Poly(A)-binding proteins modulates the stability and translation of GNRH1 mRNA in mammalian puberty. Nucleic Acids Res. 49, 3796-3813. https://doi.org/10.1093/nar/gkab155 (2021).

\section{Author contributions}

(I) Conception and design (W.D., S.L.); (II) administrative support (Y.H., Y.C. and Y.L.); (III) provision of study materials or patients (L.H., C.F.); (IV) collection and assembly of data (W.D., Y.C.); (V) data analysis and interpretation (S.L., M.L. and Z.S.); (VI) manuscript writing (all authors); (VII) final approval of manuscript (all authors).

\section{Funding}

This study was partly supported by grants from the National Natural Science Foundation of China (Nos. 81960335, 82060426), Yunnan Health Training Project of High Level Talents (No. H-2018025), the Science and Technology Department of Yunnan Province, and Kunming Medical University Joint Special Project (No. 2018FE001-070).

\section{Competing interests}

The authors declare no competing interests.

\section{Additional information}

Supplementary Information The online version contains supplementary material available at https:/doi.org/ 10.1038/s41598-021-96385-6.

Correspondence and requests for materials should be addressed to Y.C. or Y.H.

Reprints and permissions information is available at www.nature.com/reprints.

Publisher's note Springer Nature remains neutral with regard to jurisdictional claims in published maps and institutional affiliations.

(c) Open Access This article is licensed under a Creative Commons Attribution 4.0 International

License, which permits use, sharing, adaptation, distribution and reproduction in any medium or format, as long as you give appropriate credit to the original author(s) and the source, provide a link to the Creative Commons licence, and indicate if changes were made. The images or other third party material in this article are included in the article's Creative Commons licence, unless indicated otherwise in a credit line to the material. If material is not included in the article's Creative Commons licence and your intended use is not permitted by statutory regulation or exceeds the permitted use, you will need to obtain permission directly from the copyright holder. To view a copy of this licence, visit http://creativecommons.org/licenses/by/4.0/.

(c) The Author(s) 2021 